Method A retrospective review of all the neonates who had PICC lines inserted from Jan2016- Jun2018 was collected from online patient data base and case notes. The online Picture Archiving and Communication System (PACS) was utilized to review all the X-rays done post procedure.

Variables including gestational age, birth weight, site of line insertion, line tip position on X-ray and its documentation in notes and formal radiology reports, any deliberate readjustment or change in line position in subsequent X-rays, duration of use, associated complications and reasons of removal were reviewed. Detailed review of the babies who suffered complications secondary to PICC line insertion was also done.

Results Total 62 PICC lines inserted in 43 babies were reviewed. The median gestational age was 27 weeks while the birth weight was $920 \mathrm{~g}$. Right arm was the most common site of insertion $(n=19,31 \%)$. The median age at line insertion was $8 d$ with $43 \%(n=27)$ lines passed during the first week of life. Median duration of use of these lines was 5 d. Only about $26 \%(n=17)$ were optimally placed in the midline veins. Subclavian vein was the most common suboptimal position for the upper limb lines $(61 \%, n=22)$ while for the lower limb was near the hip joint $(n=7,23 \%)$ or the left side of spine with potential risk of involvement of ascending lumber $\operatorname{vein}(n=5,22 \%)$. About $29 \% \quad(n=18)$ of the lines were readjusted, amongst which the line tip within the cardiac silhouette being the most common cause. None of the lines with tips potentially in the ascending lumber vein were readjusted or further investigated. The most common complication leading to early removal was line occlusion $(\mathrm{n}=8,13 \%)$.

Discussion Malposition of the PICC tips is common and may result in significant morbidity if not recognized. Recognition of malposition, other than intracardiac, is low, and additional education is required. Additional imaging is required where tip position is unclear.

\section{GP266 A RETROSPECTIVE AUDIT OF THE INCIDENCE OF HYPOGLYCAEMIA IN AT RISK NEONATES AND COMPLIANCE WITH LOCAL GUIDELINES}

Susan Keogh*, David Corcoran. Rotunda Hospital, Dublin, Ireland

\subsection{6/archdischild-2019-epa.325}

Introduction Neonatal hypoglycaemia is a common neonatal problem and there is active debate surrounding screening, associated factors and long term consequences. There is controversy surrounding the outcome of transient neonatal hypoglycaemia but Kaiser et al reported decreased proficiency in literacy and mathematics tests in these infants at follow-up, even when adjusted for gestational age. In 2011, the American Academy of Paediatrics revised the neonatal hypoglycaemia guideline, this outlined the infants at risk for hypoglycaemia i. e large for gestational age, small for gestational age, IUGR, late preterm and maternal gestational diabetes. We have adopted a similar national and local guideline for screening and management of neonatal hypoglycaemia in Ireland.

Methods We evaluated the presence of hypoglycaemia $<3$ $\mathrm{mmol} / \mathrm{l}$ in our neonatal population over a three month period. We did this using the results from the point of care blood glucose machine. We then performed a retrospective review using the NM-CMS system to view patient records for the neonate and their mother. We were able to determine whether there were any neonatal or maternal factors that caused increased risk of hypoglycaemia.

Results We were able to retrieve data on 86 episodes of hypoglycaemia in this time period. Which occurred in 55 neonates. Compliance with guideline Only $6(10.9 \%)$ had a serum glucose sent to the lab, 49 (89\%) had three pre feed glucose measurements taken prior to discharge. 23 (41.8\%) were appropriately managed with glucogel.

Risk factors We identified $8(14.5 \%)$ of infants that were late preterm, 9 (17.6\%) infants were small for gestational age, 6 $(10.9 \%)$ large for gestational age, 25(45\%) maternal diabetes, $18(32 \%)$ increased maternal BMI, 7 (12.7\%) maternal beta blocker use.

$43(78 \%)$ of the infants identified were exclusively breastfeeding, $8(14.5 \%)$ were exclusively formula feeding. and the remaining $4(7.2 \%)$ were combination feeding.40 (72.7\%) mothers were over 35 years with $34.5 \%$ over 40 years.

Conclusion This audit confirms that hypoglycaemia is prevalent in our population, however it was not possible to identify all the hypoglycaemia episodes due to inaccurate recording on the care blood glucose machine so we have likely under estimated the true prevalence. Two of the most significant risk factors we have identified are advanced maternal age and exclusive breast-feeding. This may indicate a high-risk cohort which should be included in the early blood glucose monitoring and early feeding support to prevent negative sequelae.

\section{GP267 PREVENTION OF GROUP B STREPTOCOCCUS INFECTION- RESULTS FROM A SCREENING PROGRAM}

Catarina Ferraz Liz*, Sara Soares, Abílio Oliveira.

10.1136/archdischild-2019-epa.326

Centro Hospitalar Tâmega e Sousa, Penafiel, Portugal

Introduction Group B Streptococcus (GBS) remains one of the leading causes of early and late onset sepsis in neonate. This bacteria is present in the gastrointestinal and urinary tract of pregnant woman which could lead to vertical transmission to the newborn.

Following the first statements of the American College of Obstetricians and Gynecologists and the American Academy of Pediatrics, the Center of Disease Control and Prevention (CDC) released the first guidelines in 1996 which were reviewed in 2002 and 2010.

In the last revision, CDC recommends universal screening of pregnant women between 35-37 weeks of gestation and intrapartum antibiotic prophylaxis for those with a positive screening. This recommendation led to a decrease in GBS early onset sepsis worldwide.

In Portugal, screening is a universal practice, performed in the last prenatal consultation.

The goal of this study was to verify the main outcomes of the GBS screening program in a Portuguese maternity.

Methods A retrospective study, including all newborns $>36$ weeks of gestation born between November 2015 and May 2016 in a Portuguese maternity was conducted. Data were collected from clinical records.

Results GBS screening was performed in 1090 mothers $(96,9 \%)$, with a positive rate of $22,7 \%(n=247)$.

In 74 cases there was no/inadequate intrapartum prophylaxis $(28,6 \%)$ 